\title{
Gender Differences and Trends of Self-Rated Health in Population Aged 25-64 years from 1988 to 2017
}

\author{
Gafarov VV ${ }^{1,2}$, Panov DO ${ }^{1,2}$, Gromova EA ${ }^{1,2}$, Krymov EA $^{1,2}$, Gagulin IV ${ }^{1,2}$ and Gafarova AV $^{1,2}$ \\ ${ }^{1}$ Institute of Internal and Preventive Medicine - branch of Institute \\ of Cytology and Genetics RAS, Novosibirsk; \\ ${ }^{*}$ Corresponding authors \\ Valery V Gafarov, Collaborative Laboratory of Epidemiology Cardiovascular \\ Diseases Siberian Branch of the Russian Academy of Medical Sciences, \\ ${ }^{2}$ Collaborative laboratory of Cardiovascular Diseases Epidemiology, Novosibirsk, Russia
} Novosibirsk

Submitted:04March2021;Accepted:10 March2021;Published: 12 March 2021

Citation: Gafarov VV, Panov DO, Gromova EA, Krymov EA, Gagulin IV and Gafarova AV. (2021). Gender Differences and Trends of Self-Rated Health in Population Aged 25-64 years from 1988 to 2017. Medical \& Clinical Research 6(3): 427-434.

\begin{abstract}
The aim: To determine gender differences in the dynamic of self-rated health in an open population of 25-64 years over a long-term period - 29 years in Russia / Siberia (Novosibirsk).

Methods: Within the framework of the screening in 1988-89 under the WHO MONICA-psychosocial (MOPSY) program $(n=1676,49.5 \%$ males, mean age 44.1 \pm 0.4 years), MOPSY screening in 1994-95 ( $n=1527,43 \%$ males, mean age 44.85 \pm 0.4 years), in 2003-2005 under the international project HAPIEE ( $n=1650,34.9 \%$ males, mean age 54.25 \pm 0.2 years), in 2013-2016 ( $n=975,43.8 \%$ males, mean age 34.5 $\pm 0,4$ years) and 2016-2017 ( $n=663,41.3 \%$ years $51.95 \pm 0.32$ years) within the framework of the budgetary theme No. AAAA-A17-117112850280-2, random representative samples of men and women in one of districts in Novosibirsk were examined. Self-rated health was assessed using the questionnaire "Knowledge and attitude towards own's health".
\end{abstract}

Results: The proportion of people with negative self-reported health in the open population was extremely high in 1988. The share of women who consider themselves healthy was 13.7\%, men - 37.9\%. Gender differences increased with age. Only 10.5\% of women and 29.7\% of men had no complaints about their health. In 2013, there was an increase in positive health estimation among males and females and a decrease in the frequency of complaints, especially in female part of the population - by 2-2.5 times. The favorable trend which began in 2013 continued in 2017, when men and women more often self-esteemed own health status as "good" compared to earlier periods of observation. The proportion of women believed that taking comprehensive care of their health rose to $15 \%$ by 2017 , equaling that of men. With the exception of 2013 , women were more likely than men to report that "taking care of their health is not enough", although by 2017 this proportion had dropped to $20 \%$.

Conclusions: Sex differences in self-rated health increase with age, but the frequency of health complaints increases proportionally in men and women. There were found favorable tendencies in the decrease in the frequency of negative assessments of health, especially in female population. Further research is required to determine the stability of this trend.

Keywords: Self-Rated Health, Gender, Trends

\section{Introduction}

The World Health Survey (WHS) conducted in 2002-04 under the auspices of WHO revealed significant gender differences in self-rated health in a population aged of 18 years and older in 59 countries. The proportion of women with negative health assessments was higher than men in all age groups. $19.9 \%$ of the female population and $16.4 \%$ of the male population are not satisfied with their health. But the frequency of reporting poor health assessments worsened equally among men and women with increasing age. The smallest difference in health assessments by gender was observed in high-income countries [1].

The severity of sex differences in self-assessment of health was influenced not only by biological factors. The analysis of 
health status in the World Health Survey also showed that some differences between men and women can be associated with social determinants, mainly the level of education and employment [1]. Behavioral factors such as smoking or alcohol consumption showed no gender differences. Fertility or mortality rates in the countries studied do not explain the gender gap in self-reported health. The strong sex differences between the ages of 40-59 may be due to menopause and underreporting among men.

Recent studies showed that the relationship between self-rated health and the age of respondents is very high. Thus, the share of women who assess their health positively decreases by $42.3 \%$ when comparing the age groups of 30-44 and 45-59 years. Among men in the same age groups, the decline is only $37 \%$. Such differences are typical for older groups (60-74 years compared to 45-59), and the gender disproportion in changes in self-esteem of health is even more obvious for the elderly population ( $75+$ years). The above data refer to 2013 but the trend towards a lower decline in self-esteem of men's health was similar in 2006 and 2010 [2].

Subjective assessments are considered good predictors of health status, but may well reflect the particular attitudes of men and women towards health problems. It is possible that men report better health status due to gender roles or socioeconomic circumstances, which may partly explain why the deterioration in self-esteem of men's health status with age is milder than that of women.

The predictive role of self-reported health is used in predicting life expectancy and assessing the risk of CVD and overall mortality $[2,3]$. When assessing their general health, men tend to reflect serious and life-threatening illnesses, while women tend to reflect both life-threatening and non-life-threatening health conditions. Thus, some researchers have suggested that different approaches to health assessment entail differences in the relationship between self-reported and gender-specific outcomes.

Our research complements these prior scientific reports. Evaluating a large number of people from the general population participating in screening over the years but with a common design, increases the generalizability and relevance required for epidemiological protocols based on research principles. Thus, the aim of our study was to explore gender differences in the dynamics of self-rated health in population aged of 25-64 years over a long-term period -29 years.

\section{Methods}

The results of our study were obtained on the basis of a survey of the male and female population living in one of the districts of Novosibirsk. The examinations were carried out within the framework of screenings 1988-89, 1994-95, 2003-2005, 2013-2016 and 2016-2017. Under the II screening of the WHO program «Multinational Monitoring of Trends and Determinants of Cardiovascular Disease - Optional Psychosocial Sybstudy» (MONICA-MOPSY) representative sample of residents aged 25-64 years was examined in 1988-1989 $(\mathrm{n}=1676,49.5 \%$ males, mean age $44.1 \pm 0.4$ years, response rate $-69.8 \%$ ) [4-6].

In frame of MOPSY screening in 1994-1995 representative sample of residents aged $25-64$ years was examined $(n=1527,43 \%$ males, mean age $-44.85 \pm 0.4$ years, response rate $-77.3 \%)$. In the course of another international project HAPIEE (Health, Alcohol and Psychosocial Factors in Eastern Europe) persons aged 45-64 were examined in 2003-2005 ( $\mathrm{n}=1650,34.9 \%$ males, mean age $54.25 \pm 0.2$ years, response rate $-66.5 \%)$ [7]

In the framework of the screening studies a random representative sample survey of the population aged 25-44 years conducted in 2013-2016 by the budget scientific research theme, Gov. Task № $01201282292(\mathrm{n}=975,43.8 \%$ males, mean age $34.5 \pm 0.4$ years, response rate $-71.5 \%$ ). Within the framework of the budget theme No. AAAA-A17-117112850280-2 a survey of persons aged 3564 was carried out in 2016-2017 $(\mathrm{n}=663,41.3 \%$ males, mean age $51.95 \pm 0.32$ years, response rate $-73.6 \%)$. The study included residents of the same district of Novosibirsk as in 1994-95, 20032005 and 2013-2016.

All samples were formed on the basis of electoral lists of citizens using a table of random numbers. A random mechanical selection procedure was used. The general survey was carried out according to the standard methods accepted in epidemiology and included in the MONICA program [6]. The methods were strictly standardized and complied with the requirements of the MONICA project protocol. Validation and processing of material according to the WHO MONICA-psychosocial program was carried out at the Information Collection Center of the MEDIS Institute in Munich, Germany (Institut für Medizinische Informatik und Systemforschung). Quality control was carried out in MONICA quality control centers: Dundee (Scotland), Prague (Czech Republic), Budapest (Hungary). The presented results were considered satisfactory.

The screening survey program included registration of sociodemographic data according to the standard epidemiological protocol of the WHO MONICA-psychosocial program: identification number, place of residence, full name, date of birth, date of registration, gender, marital status, educational level, professional status. Indicators of awareness about own health and self-assessment were studied using the "Knowledge and Attitude to Own's Health" scale proposed by the MOPSY protocol and adapted to the studied population [8]. The subjects were asked to answer the questions of the scale themselves according to the instructions placed on the scale. Individuals who did not complete the questionnaire were not included in the analysis.

Statistical analysis was performed using the SPSS software package version 11.5. The study participants were standardized by age groups in the analysis. To compare the indicators between screenings, the corresponding age groups were used. To check the statistical significance of differences between groups, we used: the chi-square test $(\chi 2)$. As a criterion of statistical significance the value of the chi-square was taken into account at a certain number of degrees of freedom. The reliability of analysis was accepted at a significance level of $\mathrm{p}<0.05$.

\section{Results}

In an open population aged of 25-64 years in 1988, the proportion of the male population who rated their current status as "healthy" (the combined indicators "excellent health", "good" and 
"healthy") was $37.9 \%$. The proportion of women aged 25-64 reporting a favorable assessment of health was 3 times less and amounted to $13.7 \%(\mathrm{p}<0.001)$. With regard to age groups, it was found that there is a linear increase in persons of both sexes who assess themselves as "not quite healthy" or "sick". But in each of the age groups men were more likely than women to report being healthy $(p<0.05)$. Only a quarter of women in the $25-34$ age group considered themselves healthy and there were more than half of "healthy" men in this group (54.4\% and $25.5 \%$ for men and women, respectively; $p<0.001$ ). In the age group 35-44 years, there is a sharp decline in the female population who consider themselves healthy - more than 2 times $(p<0.001)$; this gap only widens with age. The proportion of men aged of 55-64 years with a favorable self-rated health was $18.1 \%(\mathrm{p}<0.05)$.

In 1994, there was an increase in the reporting of favorable assessments of their own health, mainly among the male population aged of 25-64 years with combined indicator "healthy" in $45.3 \%$ of men. Well rated of health in women did not increase significantly and amounted to $17.9 \%$, maintaining a 3 -fold gender gap in good self-esteem $(\mathrm{p}<0.001)$. The structure of self-rated health in age groups was similar to that in 1988. In 1994, the proportion of 55-64-year men with a good assessment of their own health was $27 \%$, which is higher than in 1988 . The proportion of people with "excellent health" was vanishingly small even in young age groups, not exceeding $2 \%$ in men and staying near zero among women, as in 1988.

The study of self-rated health in older age groups in 2003-2005 showed a steady trend in the decrease in the proportion of men who consider themselves "sick". Men 45-54 and 55-64 years with self-esteem "sick" were: 1988 - 12.7\% and 27.9\%, $1994-11.9 \%$ and $13.8 \%, 2003-8.9 \%$ and $8.8 \%$ (p for all $<0.05$ ). In women this tendency was typical only for the oldest age group while in the 4554 age group there was an increase in the proportion of people with the assessment of "sick" in comparison with 1994, returning to the 1988 level $(15.2 \%$ for $2003 ; p<0.001)$. In total, the proportion of women aged 45-54 and 55-64 years with negative self-esteem "not quite healthy" and "sick" was identical to 1988, slightly differing in the distribution of answers for these two indicators.

Two of thirds in male and female population aged 25-34 years considered themselves healthy in 2013-2016. In this age group sex differences in the combined indicator "healthy" were almost completely offset. The proportion of people aged 35-44 years who consider themselves "healthy" was also significantly higher than in 1988 and 1994 and amounted to $58.4 \%$ for the male and $40.7 \%$ for the female part of the population $(\mathrm{p}<0.001)$.

In 2017, the trend of an increase in positive assessments of health among men and women in the open population aged 35-64 continued. Thus, the proportion of men and women aged of 35-44 years with the combined indicator "healthy" was the maximum for the entire 29 -year observation period and amounted to $71.8 \%$ and $64.3 \%$, respectively. The gender difference in the responses of this age group was significantly lower than in the previous screenings in 2013-2016, 1994 and 1988 and was not statistically significant. But in older age groups, gender differences in self-rated health were obvious, reaching 17.8\% (a pooled answer is "not quite healthy" and "sick") in the 45-54 age group (p for all $<0.05$ ). Despite the fact that older women showed significantly worse self-rated health than men, the proportion of "not quite healthy" and "sick" in the 45-54 and 55-64 age groups was $20-30 \%$ lower than in the period from 1988 to $2003-05$. 
Table 1: Gender differences in trends of self-rated health in population of 25-64 years depending on age

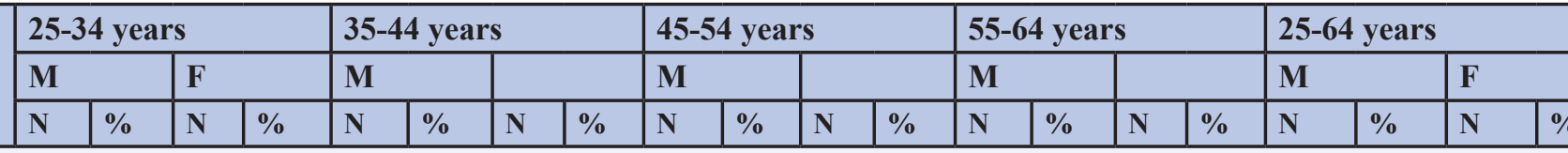

How do you rate your state of health?

\begin{tabular}{|c|c|c|c|c|c|c|c|c|c|c|c|c|c|c|c|c|c|c|c|c|c|}
\hline Excellent health & \multirow[t]{6}{*}{1988} & 4 & 2.0 & 1 & 0.5 & 3 & 1.5 & 0 & $\mathbf{0}$ & 2 & 1.2 & 0 & $\mathbf{0}$ & 2 & 1.3 & 2 & 1.4 & 11 & 1.5 & 3 & 0.4 \\
\hline Good & & 28 & 13.7 & 8 & 4.3 & 11 & 5.5 & 5 & 2.4 & 9 & 5.2 & 3 & 1.6 & 7 & 4.5 & 1 & 0.7 & 56 & 7.6 & 17 & 2.3 \\
\hline Healthy & & 79 & 38.7 & 38 & 20.5 & 73 & 36.3 & 19 & 9.0 & 38 & 22.0 & 15 & 8.1 & 19 & 12.3 & 7 & 5.1 & 213 & 28.8 & 80 & 11.0 \\
\hline Not quite healthy & & 86 & 42.2 & 127 & 68.6 & 97 & 48.3 & 167 & 78.8 & 102 & 59.0 & 140 & 75.3 & 83 & 53.9 & 89 & 64.5 & 369 & 49.9 & 530 & 72.6 \\
\hline Sick & & 7 & 3.4 & 11 & 5.9 & 17 & 8.5 & 21 & 9.9 & 22 & 12.7 & 28 & 15.1 & 43 & 27.9 & 39 & 28.3 & 90 & 12.2 & 100 & 13.7 \\
\hline \multirow[t]{2}{*}{ Total } & & 204 & 100 & 185 & 100 & 201 & 100 & 212 & 100 & 173 & 100 & 186 & 100 & 154 & 100 & 138 & 100 & 739 & 100 & 730 & 100 \\
\hline & & \multicolumn{4}{|c|}{$\chi^{2}=35.216 \mathrm{df}=4 \mathrm{p}<0.001$} & \multicolumn{4}{|c|}{$\chi^{2}=55.647 \mathrm{df}=4 \mathrm{p}<0.001$} & \multicolumn{4}{|c|}{$\chi^{2}=21.225 \mathrm{df}=4 \mathrm{p}<0.001$} & \multicolumn{4}{|c|}{$\chi^{2}=9.595 \mathrm{df}=4 \mathrm{p}<0.05$} & \multicolumn{4}{|c|}{$\chi^{2}=187.354 \mathrm{df}=4 \mathrm{p}<0.001$} \\
\hline Excellent health & \multirow[t]{6}{*}{1994} & 3 & 1.8 & 0 & $\mathbf{0}$ & 1 & 0.6 & 1 & 0.6 & 1 & 0.7 & 0 & $\mathbf{0}$ & 3 & 1.7 & 1 & 1.4 & 8 & 1.2 & 2 & 0.5 \\
\hline Good & & 33 & 19.4 & 8 & 6.3 & 28 & 15.9 & 6 & 3.9 & 9 & 6.7 & 1 & 2.2 & 9 & 5.2 & 0 & $\mathbf{0}$ & 79 & 12.1 & 15 & 3.8 \\
\hline Healthy & & 80 & 45.9 & 27 & 21.3 & 61 & 34.7 & 17 & 11.0 & 35 & 26.1 & 5 & 11.1 & 34 & 20.1 & 5 & 7.1 & 210 & 32 & 54 & 13.6 \\
\hline Not quite healthy & & 54 & 30.8 & 82 & 64.6 & 78 & 44.3 & 106 & 68.8 & 74 & 54.5 & 36 & 80.0 & 100 & 59.2 & 50 & 71.4 & 306 & 46.6 & 274 & 69.2 \\
\hline Sick & & 5 & 2.9 & 10 & 7.9 & 9 & 4.5 & 24 & 15.6 & 17 & 11.9 & 3 & 6.7 & 23 & 13.8 & 14 & 20.0 & 54 & 8.1 & 51 & 12.9 \\
\hline \multirow[t]{2}{*}{ Total } & & 175 & 100 & 127 & 100 & 177 & 100 & 154 & 100 & 136 & 100 & 45 & 100 & 169 & 100 & 70 & 100 & 657 & 100 & 396 & 100 \\
\hline & & \multicolumn{4}{|c|}{$\chi^{2}=45.447 \mathrm{df}=4 \mathrm{p}<0.001$} & \multicolumn{4}{|c|}{$\chi^{2}=48.772 \mathrm{df}=4 \mathrm{p}<0.001$} & \multicolumn{4}{|c|}{$\chi^{2}=9.469 \mathrm{df}=4 \mathrm{p}=0.05$} & \multicolumn{4}{|c|}{$\chi^{2}=11.361 \mathrm{df}=4 \mathrm{p}<0.05$} & \multicolumn{4}{|c|}{$\chi^{2}=81.524 \mathrm{df}=4 \mathrm{p}<0.001$} \\
\hline Good & \multirow{5}{*}{2003} & & & & & & & & & 23 & 7.6 & 8 & 1.4 & 7 & 2.6 & 11 & 2.1 & 30 & 5.2 & 19 & 1.8 \\
\hline Healthy & & & & & & & & & & 79 & 26.0 & 53 & 9.6 & 57 & 21.0 & 27 & 5.2 & 136 & 23.6 & 80 & 7.4 \\
\hline Not quite healthy & & & & & & & & & & 172 & 56.6 & 408 & 73.6 & 180 & 66.2 & 398 & 76.5 & 352 & 61.1 & 806 & 75.0 \\
\hline Sick & & & & & & & & & & 27 & 8.9 & 84 & 15.2 & 24 & 8.8 & 81 & 15.6 & 51 & 8.9 & 165 & 15.4 \\
\hline \multirow[t]{2}{*}{ Total } & & & & & & & & & & 304 & 100 & 554 & 100 & 272 & 100 & 520 & 100 & 576 & 100 & 1074 & 100 \\
\hline & & & & & & & & & & \multicolumn{4}{|c|}{$\chi^{2}=71.941 \mathrm{df}=4 \mathrm{p}<0.001$} & \multicolumn{4}{|c|}{$\chi^{2}=52.391 \mathrm{df}=4 \mathrm{p}<0.001$} & \multicolumn{4}{|c|}{$\chi^{2}=116.25 \mathrm{df}=4 \mathrm{p}<0.001$} \\
\hline Excellent health & \multirow[t]{6}{*}{2013} & 9 & 5.5 & 5 & 2.3 & 5 & 1.9 & 4 & 1.2 & & & & & & & & & 14 & 3.3 & 9 & 1.6 \\
\hline Good & & 39 & 23.6 & 63 & 29.6 & 48 & 18.3 & 53 & 15.9 & & & & & & & & & 87 & 20.4 & 116 & 21.2 \\
\hline Healthy & & 68 & 41.2 & 73 & 34.3 & 100 & 38.2 & 79 & 23.7 & & & & & & & & & 168 & 39.3 & 152 & 27.8 \\
\hline Not quite healthy & & 46 & 27.9 & 71 & 33.3 & 103 & 39.3 & 185 & 55.4 & & & & & & & & & 149 & 34.9 & 256 & 46.8 \\
\hline Sick & & 3 & 1.8 & 1 & 0.5 & 6 & 2.3 & 13 & 3.9 & & & & & & & & & 9 & 2.1 & 14 & 2.6 \\
\hline \multirow[t]{2}{*}{ Total } & & 165 & 100 & 213 & 100 & 262 & 100 & 334 & 100 & & & & & & & & & 427 & 100 & 547 & 100 \\
\hline & & n.s. & & & & $\chi^{2}=2$ & $374 d$ & $=4 \mathrm{p}$ & 0.001 & & & & & & & & & $\chi^{2}=20$ & $19 \mathrm{df}=$ & $4 \mathrm{p}<0$. & \\
\hline Good & & & & & & 9 & 12.7 & 18 & 18.4 & 7 & 8.5 & 12 & 8.7 & 11 & 9.1 & 8 & 5.3 & 27 & 9.9 & 38 & 9.8 \\
\hline Healthy & & & & & & 40 & 56.3 & 43 & 43.9 & 48 & 58.5 & 56 & 40.6 & 49 & 40.5 & 39 & 25.7 & 137 & 50.0 & 138 & 35.6 \\
\hline Not quite healthy & & & & & & 20 & 28.2 & 33 & 33.7 & 26 & 31.7 & 70 & 50.7 & 58 & 47.9 & 95 & 62.5 & 104 & 38.0 & 198 & 51.0 \\
\hline Sick & & & & & & 0 & 0 & 2 & 2.0 & 1 & 1.2 & 0 & 0 & 3 & 2.5 & 8 & 5.3 & 4 & 1.5 & 10 & 2.6 \\
\hline Total & & & & & & 71 & 100 & 98 & 100 & 82 & 100 & 138 & 100 & 121 & 100 & 152 & 100 & 274 & 100 & 388 & 100 \\
\hline & & & & & & n.s. & & & & $\chi^{2}=9$ & $456 \mathrm{df}=$ & $=4 p<0$ & & $\chi^{2}=11$ & $.458 \mathrm{~d}$ & $=4 \mathrm{p}<$ & 0.05 & $\chi^{2}=15$ & $18 \mathrm{df}=4$ & $\mathrm{p}<0.0$ & \\
\hline
\end{tabular}

To the question: "Do you have any complaints about your health?" $70 \%$ of the male and almost $90 \%$ of the female population of 25 64 years answered "Yes" in 1988. In contrast to self-rated health where there was a linear increase in unfavorable assessments the maximum number of complaints about health was observed among men and women in the 45-54 age group (81\% and 94.9\%, respectively; $\mathrm{p}<0.001)$. Even in the youngest age group, $25-44$ years, the proportion of women without complaints was less than $20 \%$; but there were 2 times more men without complaints in this group $(40.1 \%$ and $18.1 \%$, respectively; $\mathrm{p}<0.001)$. 
In 2003-2005, the frequency of health complaints among men and women decreased in the $45-54$ age group by $10 \%$ and $5 \%$, respectively; but in the group of 55-64 years it did not change significantly. It slightly increased in men by $1.5 \%$ in comparison with 1988 ( for all $<0.001$ ). In 2013-2016, the share of persons who did not complain about health increased significantly mainly due to the female part of the population - by 2-2.5 times in the age groups of 25-44 and 35-44 years, in comparison with 1988 and 1994. Sex differences in the structure of responses disappeared in the 25-34-year group.
More than half of men and a third of women in the open population aged 35-64 had no health complaints in 2016-2017 ( $\mathrm{p}<0.001$ ). $71.8 \%$ of men and $52 \%$ of women aged $35-44$ years had no complaints $(p<0.05)$. These are the best indicators in comparison with the previous periods. At the same time the trend in the increase of a gender gap in comparison with 2013 was again found. This difference is even more pronounced in the oldest age group of men without complaints about health who had an almost 2-fold gap from women $(47.9 \%$ and $25 \%$, for men and women aged 55-64 years, respectively; $\mathrm{p}<0.001)$.

Table 2: Gender differences in trends of self-rated health in population of 25-64 years depending on age

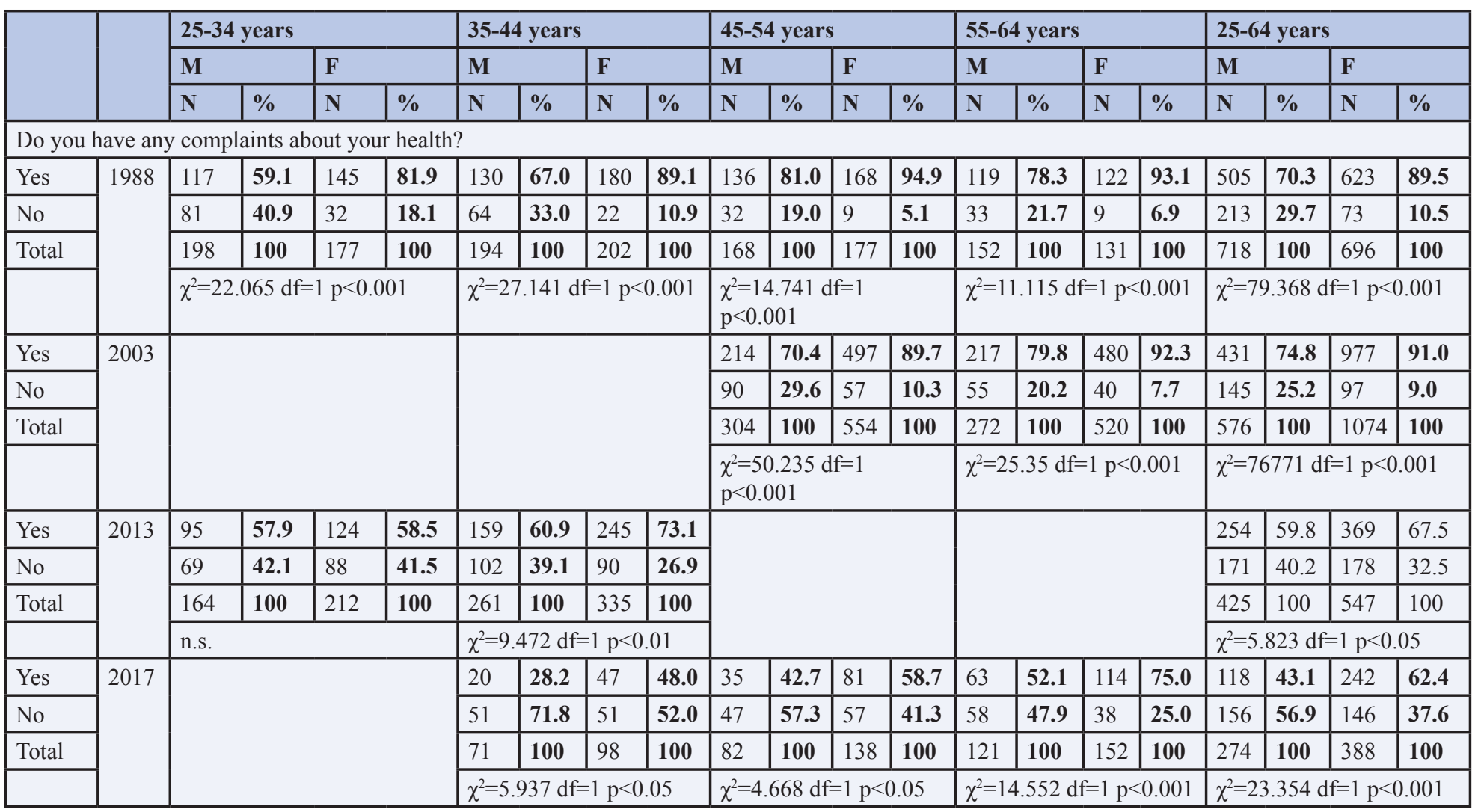

Less than $10 \%$ of men and only $6.1 \%$ of women in general population aged 25-64 in 1988 took comprehensive care of their health based on their own perceptions $(\mathrm{p}<0.001)$. There has been an increase in health-conscious men and women with age. Men aged of 55-64 years more often showed concern for their health in comparison with women of the same age group, although these differences did not reach statistical significance. More than half of male and female population aged 25-64 years believed that taking care of their health is not enough. This opinion was most clearly observed among women $45-54$ years, reaching $68.1 \%$. In this age group gender differences in responses were stronger $(p<0.05)$. Younger age groups are more likely to believe that they "could take more care of their health" $(52.9 \%$ and $43.5 \%$ for men and women 25-34 years old; n.s.).

In 1994, women in the youngest age group were more likely to state that they "could take more care of their health". The structure of the responses was similar to 1988 in total but there were some differences. The proportion of women believed that taking care of their health is not enough was higher in the 35-44 age group; this indicates a shift towards younger age groups of women compared to 1988 .

In 2003-2005, there was a tendency towards a decrease in the proportion of persons of both sexes who "takes care of their health not enough" ( $42.5 \%$ and $48.4 \%$ for men and women, respectively; $\mathrm{p}=0.056$ ). This decline was evident in women aged of 45-54 years. In dynamics, the number of men 45-54 and 55-64 years who "care about health" has decreased by $5-8 \%$. But, as in 1988, men aged 55-64 were more likely to indicate that they "care enough about their health" than women (11\% and 9\% for men and women, respectively; n.s.). In 2003, there was an increase in the proportion of study participants who believed that they "could take more care of their health" without significant gender differences in age groups. 
An upward trend in the proportion of people who care about health in population aged 25-44 years was found in 2013-2016, especially in the group of women aged 25-34, increasing the gender gap in this age group ( $13.4 \%$ and $23 \%$ for men and women, respectively; $\mathrm{p}=0.054)$. The proportion of those who "does not care enough about their health" was several times less than in 1988; it was more evident in women of the youngest age $(51.6 \%, 57.5 \%$ and $9.9 \%$, for 1988, 1994 and 2013, respectively).

In 2016-2017, the proportion of participants who believe that taking care of their health is comprehensive was the highest for the entire 29-year observation period and amounted to $15.7 \%$ and $14.9 \%$ for the male and female population 35-64 years, respectively. Men more often than women stated that they take care of their health in the 35-44 and 45-54 age groups $(\mathrm{p}<0.05)$. A further decrease in the proportion of men and women who reported that taking care of their health is not enough in comparison with the previous screening in 2013-2016 was found in the 35-44 age group: $28.4 \%$ and $14.1 \%$ of men, $26.9 \%$ and $19.4 \%$ of women for 2013 and 2017, respectively. This downward trend was associated with an increase in the number of respondents in all age groups who believed that they "could take more care of their health". In age groups 35-44 and 55-64 years, men more often than women reported that they "could take more care of their health": $71.8 \%$ and $67.3 \%, 67.8 \%$ and $45.8 \%$, for men and women, respectively; $\mathrm{p}$ for all $<0.05)$.

Table 3: Gender differences in trends of self-rated health in population of 25-64 years depending on age

\begin{tabular}{|c|c|c|c|c|c|c|c|c|c|c|c|c|c|c|c|c|c|c|c|c|c|}
\hline & & \multicolumn{4}{|c|}{ 25-34 years } & \multicolumn{4}{|c|}{$35-44$ years } & \multicolumn{4}{|c|}{ 45-54 years } & \multicolumn{4}{|c|}{ 55-64 years } & \multicolumn{4}{|c|}{ 25-64 years } \\
\hline & & \multicolumn{2}{|l|}{$\mathbf{M}$} & \multicolumn{2}{|l|}{$\mathbf{F}$} & \multicolumn{2}{|l|}{$\mathbf{M}$} & \multicolumn{2}{|l|}{$\mathbf{F}$} & \multicolumn{2}{|l|}{$\mathbf{M}$} & \multicolumn{2}{|l|}{ M } & \multicolumn{2}{|l|}{$\mathbf{F}$} & \multicolumn{2}{|l|}{$\mathbf{M}$} & \multicolumn{2}{|l|}{$\mathbf{F}$} & \multicolumn{2}{|l|}{$\mathbf{F}$} \\
\hline & & $\mathbf{N}$ & $\%$ & $\mathbf{N}$ & $\%$ & $\mathbf{N}$ & $\%$ & $\mathbf{N}$ & $\%$ & $\mathbf{N}$ & $\%$ & $\mathbf{N}$ & $\%$ & $\mathbf{N}$ & $\%$ & $\mathbf{N}$ & $\%$ & $\mathbf{N}$ & $\%$ & $\mathbf{N}$ & $\%$ \\
\hline \multicolumn{22}{|c|}{ Do you believe that taking care of your health is comprehensive? } \\
\hline Yes & \multirow[t]{5}{*}{1988} & 12 & 5.8 & 9 & 4.9 & 12 & 6.0 & 11 & 5.2 & 18 & 10.3 & 10 & 5.4 & 29 & 19.1 & 18 & 13.0 & 71 & 9.6 & 49 & 6.7 \\
\hline Could take more care & & 108 & 52.9 & 80 & 43.5 & 75 & 37.5 & 63 & 29.9 & 60 & 34.5 & 49 & 26.5 & 50 & 32.9 & 41 & 29.7 & 293 & 39.8 & 235 & 32.3 \\
\hline It is not enough & & 84 & 41.2 & 95 & 51.6 & 113 & 56.5 & 137 & 64.9 & 96 & 55.2 & 126 & 68.1 & 73 & 48.0 & 79 & 57.2 & 373 & 50.6 & 443 & 61.0 \\
\hline Total & & 204 & 100 & 184 & 100 & 200 & 100 & 211 & 100 & 174 & 100 & 185 & 100 & 152 & 100 & 138 & 100 & 737 & 100 & 727 & 100 \\
\hline & & \multicolumn{4}{|l|}{ n.s. } & \multicolumn{4}{|l|}{ n.s. } & \multicolumn{4}{|c|}{$\chi^{2}=7.119 \mathrm{df}=2 \mathrm{p}<0.05$} & \multicolumn{4}{|l|}{ n.s. } & \multicolumn{4}{|c|}{$\chi^{2}=16.342 \mathrm{df}=2 \mathrm{p}<0.001$} \\
\hline Yes & \multirow[t]{4}{*}{1994} & - & - & 5 & 3.9 & - & - & 7 & 4.5 & - & - & 3 & 6.7 & - & - & 8 & 11.1 & - & - & 23 & 5.7 \\
\hline Could take more care & & - & - & 49 & 38.6 & - & - & 35 & 22.3 & - & - & 10 & 22.2 & - & - & 13 & 18.1 & - & - & 107 & 26.7 \\
\hline It is not enough & & - & - & 73 & 57.5 & - & - & 115 & 73.2 & - & - & 32 & 71.1 & - & - & 51 & 70.8 & - & - & 271 & 67.6 \\
\hline Total & & - & - & 127 & 100 & - & - & 157 & 100 & - & - & 45 & 100 & - & - & 72 & 100 & - & - & 401 & 100 \\
\hline Yes & \multirow[t]{5}{*}{2003} & & & & & & & & & 16 & 5.3 & 40 & 7.2 & 30 & 11.0 & 47 & 9.0 & 46 & 8.0 & 87 & 8.1 \\
\hline Could take more care & & & & & & & & & & 148 & 48.7 & 236 & 42.6 & 137 & 50.4 & 231 & 44.4 & 285 & 49.5 & 467 & 43.5 \\
\hline It is not enough & & & & & & & & & & 140 & 46.1 & 278 & 50.2 & 105 & 38.6 & 242 & 46.5 & 245 & 42.5 & 520 & 48.4 \\
\hline Total & & & & & & & & & & 304 & 100 & 554 & 100 & 272 & 100 & 520 & 100 & 576 & 100 & 1074 & 100 \\
\hline & & & & & & & & & & n.s. & & & & $\chi^{2}=4$ & $53 \mathrm{df}=$ & $\mathrm{p}=0.0$ & & $\chi^{2}=5$ & $763 \mathrm{df}=$ & $=2 \mathrm{p}=0$ & 056 \\
\hline Yes & 2013 & 22 & 13.4 & 49 & 23.0 & 25 & 9.5 & 44 & 13.1 & & & & & & & & & 47 & 11.1 & 93 & $\mathbf{1 7 . 0}$ \\
\hline Could take more care & & 121 & 73.8 & 143 & 67.1 & 162 & 62.1 & 201 & 60.0 & & & & & & & & & 283 & 66.6 & 344 & 62.8 \\
\hline It is not enough & & 21 & 12.8 & 21 & 9.9 & 74 & 28.4 & 90 & 26.9 & & & & & & & & & 95 & 22.4 & 111 & 20.3 \\
\hline Total & & 164 & 100 & 213 & 100 & 261 & 100 & 335 & 100 & & & & & & & & & 425 & 100 & 548 & 100 \\
\hline & & $\chi^{2}=5$ & $831 \mathrm{df}$ & $=2 \mathrm{p}=0$ & 0.054 & n.s. & & & & & & & & & & & & $\chi^{2}=6$ & $352 \mathrm{df}=$ & $=2 \mathrm{p}<0$ & \\
\hline Yes & 2017 & & & & & 10 & 14.1 & 13 & 13.3 & 16 & 19.5 & 16 & 11.6 & 17 & 14.0 & 29 & 19.0 & 43 & 15.7 & 58 & 14.9 \\
\hline Could take more care & & & & & & 51 & 71.8 & 66 & 67.3 & 45 & 54.9 & 100 & 72.5 & 82 & 67.8 & 70 & 45.8 & 178 & 65.0 & 236 & 60.7 \\
\hline It is not enough & & & & & & 10 & 14.1 & 19 & 19.4 & 21 & 25.6 & 22 & 15.9 & 22 & 18.2 & 54 & 35.3 & 53 & 19.3 & 95 & 24.4 \\
\hline Total & & & & & & 71 & 100 & 98 & 100 & 82 & 100 & 138 & 100 & 121 & 100 & 153 & 100 & 274 & 100 & 389 & 100 \\
\hline & & & & & & $\chi^{2}=2$ & 7.943 & $\mathrm{df}=2 \mathrm{p}$ & 0.05 & $\chi^{2}=7$ & $.09 \mathrm{df}=$ & $2 \mathrm{p}<0$ & & $\chi^{2}=1$ & $.005 \mathrm{~d}$ & $2 \mathrm{p}<0$ & .001 & n.s. & & & \\
\hline
\end{tabular}

\section{Discussion}

The proportion of people in general population with negative self-rated health is extremely high, especially during periods of socioeconomic disadvantage. These are the years of USSR ends and the early post-Soviet period. Men are significantly ahead of women in the frequency of favorable health assessments. Whereas more than half of the women in our study consider themselves not entirely health or ill. In other studies, women are more likely than men to be dissatisfied with their health $(20.9 \%$ and $16.9 \%)$. Quite understandably the proportion of people dissatisfied with their 
health is higher among the older age groups of 45-64 years [7]. In 1994, the proportion of men aged of 55-64 years with a good assessment own's health was $27 \%$ - this was higher than in 1988 , which did not affect, in general, the low prevalence of good selfrated health in Russia, in comparison with other countries [1-3].

Despite the fact that only $18.1 \%$ of the youngest age group had no complaints about health in 1988, the proportion of women who consider themselves healthy was slightly higher $-25.5 \%$. In men, this difference with self-rated health is even higher: $40.1 \%$ had no complaints about their health but $54.4 \%$ considered themselves healthy. This indicates that health complaints and self-esteem are not identical. Probably, the awareness of oneself as a sick person requires confirmation, for example, additional diagnostic procedures compared to the presence of health complaints at the moment. This justifies the frequent opinion of the respondents in our studies that they will not immediately believe the doctor but will do a number of additional tests [6].

2013 can be called a turning point with the increase in a favorable assessment of health when in male and female population and a decrease in the frequency of complaints, especially among the female part of the population - by 2-2.5 times. It should be noted that gender differences in the reporting of complaints and selfrated health were present throughout the entire study - 29 years.

The favorable trend, which began in 2013, continued in 2017, when men and women reported more often that they were "healthy", compared to earlier periods of observation. Based on unpublished data on the growth trend of negative psychological characteristics in 2016-2017, such as anxiety, depression, life exhaustion - these affective states probably have not yet altered perceptions of own health status. But future screening studies of this population may change current trends.

Self-rated health and related factors were investigated in a sample of women aged 18-70 years living in a city in Saudi Arabia. Data was collected from July to September 2015 using a questionnaire from randomly selected shopping mall visitors. An unfavorable health assessment is often associated with 2 or more diseases. Persons with insufficient physical activity, overweight, living or working in unfavorable environmental conditions, as well as persons with unfavorable psychosocial health, more often report a negative assessment of health [8].

The use of a large database of the European Working Conditions Survey 2010 (30124 observations) revealed certain patterns in the reporting by women of a relatively worse health assessment. It found that in a professional environment where women are the majority, participants tend to report worse health conditions than those employed in a male-dominated team, regardless of gender. These results are robust when adjusted for a large number of professional indicators. And this is not due to the lower quality of work in a team dominated by women. Such data indicate that social norms associated with a particular gender environment play an important role in explaining differences in health-related behavior among different genders, at least in the workplace [9].

Self-rated health and lifestyle behavior are related with socioeconomic circumstances. However, a number of researchers recognize that the level of education influences psychosocial factors to a greater extent than the level of income [10].

Psychological characteristics also play the role of mediators influencing the state of health. Lack of social support and social isolation or loneliness are associated with cardiovascular health in both sexes. However, metabolic effects and the incidence of diabetes were only seen in women but not in men. Moreover, stress, lack of physical activity and sleep disturbances were found to be important mediators in these associations, especially among the elderly [11].

In our study, men surprisingly more often than women of the same age believed that "take comprehensive care of their health" in 1988. In dynamics, the proportion of women believed that taking care of their health is comprehensive has reached $15 \%$, catching up with men. But there were fewer ones than those who pay insufficient attention to health both among men and women. With the exception of 2013, women are more likely than men to believe that taking care of their health is not enough. Since 1988 to 2017 the proportion of persons who "take care of their health not enough" had decreased from $50 \%$ in men and $60 \%$ in women to $20 \%$ in both sexes aged $25-64$ years.

Recent studies showed women are more likely than men to comply with measures related to primary prevention [12]. These differences were found in following areas: collection of health information, hygiene, sexual safety, sun protection. In terms of lifestyle modification, men showed greater commitment to physical activity and active leisure time. Prevention of cardiometabolic diseases by affecting hemodynamic parameters, lipids and inflammation was not associated with the presence of gender differences. Despite the fact that the use of health care resources in relation to mental health did not differ between men and women, some researchers indicate their lower use among men [12].

\section{Conclusions}

1. The proportion of people with negative self-rated health is extremely high in an open population in 1988. Despite the fact that only $10.5 \%$ had no health complaints in 1988 , the proportion of women who considered themselves healthy was slightly higher $-13.7 \%$. In men this difference with self-rated health is even higher: $29.7 \%$ of men had no complaints about their health but $37.9 \%$ rated their health status as "good".

2. In 2013, there was an increase in positive health estimation among male and female population and a decrease in the frequency of complaints, especially among the female part of the population - by 2-2.5 times. The favorable trend which began in 2013 continued in 2017, when men and women more often considered own health status as "good" compared to earlier periods of observation.

3. Sex differences in the reporting of complaints and self-rated health were present throughout the 29-year study; this gap only widens with age. Men are significantly ahead of women in the frequency of favorable health assessments.

4. The proportion of women believed that taking care of their health is comprehensive rose to $15 \%$ by 2017 , equaling that of 
men. With the exception of 2013, women are more likely than men to indicate that "taking care of their health is not enough", although by 2017 this proportion had dropped to $20 \%$.

\section{References}

1. Boerma T, Hosseinpoor AR, Verdes E (2016) A global assessment of the gender gap in self-reported health with survey data from 59 countries. BMC Public Health 16: 675.

2. Łyszczarz B (2017) Gender bias and sex-based differences in health care efficiency in Polish regions. Int J Equity Health 16: 8 .

3. Ryou I, Cho Y, Yoon H-J, Park M (2019) Gender differences in the effect of self-rated health (SRH) on all-cause mortality and specific causes of mortality among individuals aged 50 years and older. PLoS ONE 14: e0225732.

4. Tunstall-Pedoe H, Kuulasmaa K, Tolonen H, Davidson M, Mendis S (2020) with 64 other contributors for The WHO MONICA Project. MONICA Monograph and Multimedia Sourcebook. World's largest study of heart disease, stroke, risk factors, and population trends 1979-2002. Edited by H. Tunstall-Pedoe. WHO: Geneve; on-line publication.

5. UCL department of epidemiology and public health Central and Eastern Europe research group HAPIEE study. http:// www.ucl.ac.uk/easteurope/hapiee-cohort.htm.

6. Gafarov VV, Panov DO, Gromova EA, Gagulin IV, Gafarova AV, et al. (2018) 23-year dynamics (1994-2016) relationships to its health, behavioral characteristics and prevention of cardiovascular diseases among women 25-44 years in Russia /Siberia. Terapevticheskii arkhiv 90: 36-44.

7. Gill TK, Price K, Dal Grande E, Daly A, Taylor AW (2016) Feeling angry about current health status: using a population survey to determine the association with demographic, health and social factors. BMC Public Health 16: 588.

8. Jradi H, Alharbi Z, Mohammad Y (2018) Self-rated Health Among Saudi Women: Association with Morbidity, Lifestyle, and Psychosocial Factors. Journal of Epidemiology and Global Health 8: 183-188.

9. Caroli E, Weber-Baghdiguian L (2016) Self-reported health and gender: The role of social norms. Soc Sci Med 153: 220229.

10. Kino S, Kawachi I (2020) How much do preventive health behaviors explain education- and income-related inequalities in health? Results of Oaxaca-Blinder decomposition analysis. Ann Epidemiol 43: 44-50.

11. Christiansen J, Larsen FB, Lasgaard M (2016) Do stress, health behavior, and sleep mediate the association between loneliness and adverse health conditions among older people? Soc Sci Med 152: 80-86.

12. Hiller J, Schatz K, Drexler H (2017) Gender influence on health and risk behavior in primary prevention: a systematic review. J Public Health 25: 339-349.
Copyright: (C2021 Valery V Gafarov, et al. This is an open-access article distributed under the terms of the Creative Commons Attribution License, which permits unrestricted use, distribution, and reproduction in any medium, provided the original author and source are credited. 